\title{
A Novel NSCT based Illuminant Invariant Extraction with Optimized Edge Detection Technique for Face Recognition
}

\author{
S.H.Krishna Veni \\ Research scholar \\ M.S University \\ Tirunelveli
}

\author{
K.LShunmuganathan \\ Professor \\ R.M.K.Engineering college \\ Chennai
}

\author{
L.Padma Suresh \\ Professor \\ $\mathrm{NICHE}$ \\ Kumaracoil
}

\begin{abstract}
ABTRACT
A novel integrated approach for resolving the effect of illumination changes on face recognition is proposed. This face recognition system comprises feature extraction, feature selection and recognition. For feature extraction, a normal shrink filter in NSCT domain denoising technique is applied which produces illuminant invariant for the given image. In the second phase, to capture the important geometrical structures and to reduce the feature dimensionality Ant colony Optimization algorithm is performed. This combined approach fairly detects the edges with improved quality. Finally for recognition, a graph matching algorithm is employed. This algorithm utilizes a group of feature points to explore their geometrical relationship in a graph arrangement. While applying the entire method to the yaleB database, experimental results shows that the proposed work yields the best subset of features and provides a better solution for complex illumination problems.
\end{abstract}

\section{Keywords:}

illuminant invariant, Non subsampled contourlet, feature subset, Ant colony optimization, Weighted graph matching

\section{INTROUCTION}

The most challenging aspects of face recognition are variations in head pose, expression and illumination. Among these factors head pose and illumination changes become a great issue. Various approaches have been proposed in the last decades to eradicate the illumination problems.

In earlier developments, techniques like logarithm transform and histogram equalization [4] were used. They work well for low illumination variant images but difficult to deal with complicated illumination variations. Dang-Hui Liu, Kin-Man Lam, proposed a method for illumination changes by using a ratio-image [7] between the face image and a reference face image, both of which are blurred by a Gaussian filter. Fixed illumination condition is possible here. Still complex illumination problems cannot be rectified completely. Young Kyung Park, Seok Lai Park, Joong Kyu Kim uses Retinex theory[8] for illumination estimation and normalization by using several low pass filters with different cut-off frequencies. LCE [5] efficiently improves visibility of the fine textures but it may result in problems of visible gradient reversal or generates undesired halos. A comparative study of illumination preprocessing methods [11] were proposed by Hu Han, Shiguang Shan, shows that holistic illumination preprocessing approaches, e.g. HE, GIC, LTV and TT, improves the performance and the face reflectance field estimation based methods decompose a face image into two bands. Two-band decomposition is still insufficient for analyzing the importance of individual feature bands in face recognition.

In recent years the development were focused on multiband decomposition of face images. Under this, multi resolution concepts were spread rapidly. Wavelet[6] transforms emerges which has better edge preserving ability in low frequency Illumination fields and better useful information saving ability in high frequency fields. But it fails to preserve the important feature extraction properties like directionality and anisotropy. Also it can only detect the edges in horizontal, vertical and $45^{\circ}$ orientations. Later a new image representation was proposed namely, the Contourlet transform which is a new two-dimensional extension of the wavelet transform using multiscale and directional filter banks. But, oversampling becomes a critical issue. Hence recent developments focus on Non subampled contourlet transfoms.

This proposed work adopts a Normal Shrinkfilter in NSCT domain [1] to extract illumination invariant. Compared with the existing methods, the proposed method can better preserve edges due to the nonsubsampled contourlet transform with multiscale, multi direction analysis and shift-invariance, The peculiar feature of the proposed work is integrating Ant colony optimization algorithm (ACO)[2][3] to the extracted illuminant invariant image. Experimental results on the YaleB database shows that the proposed method is robust and effective for face recognition with varying illumination conditions.

\section{METHODOLOGY}

The methodology of implementation is organized as follows: Section 2.1 explains about preprocessing method. Section 2.2 describes the NSCT-based method for illumination invariant extraction. In section 2.3, ACO algorithm is presented and in section2.4 graph matching algorithm is discussed. Experimental results are discussed in section 3. Finally, section 4 deals with conclusions and future work. The Data flow diagram for the proposed method is given in fig 1 .

\subsection{Preprocessing}

A gray scale face image $\mathrm{F}$ under illumination conditions is represented by,

$\mathrm{F}(\mathrm{x}, \mathrm{y})=\mathrm{I}(\mathrm{x}, \mathrm{y}) \cdot \mathrm{R}(\mathrm{x}, \mathrm{y})$

where $I(x, y)$ and $R(x, y)$ are the illumination and the reflectance at a point $(\mathrm{x}, \mathrm{y})$. The value of $\mathrm{R}$ cannot be obtained directly, hence a Normal Shrink-based denoising model is 
applied to extract R. To apply denoising model, logarithmic transform is computed.

Thus, Eq.(1) can be rewritten as

$\mathrm{F}^{\prime}=\mathrm{I}^{\prime}+\mathrm{R}^{\prime}$

The transformation of this type, expand the values of dark pixels in an image while compressing the higher-level values. As a result, logarithm transform can partially reduce the effect of various illumination conditions. Fig. 2(a) and 2(b) shows the original and logarithm image of low illuminant variant image.

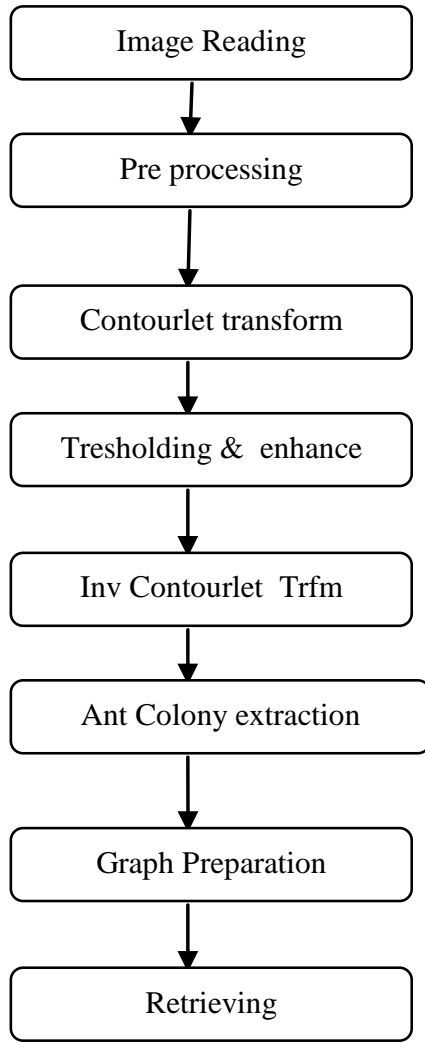

Fig.1 data flow diagram

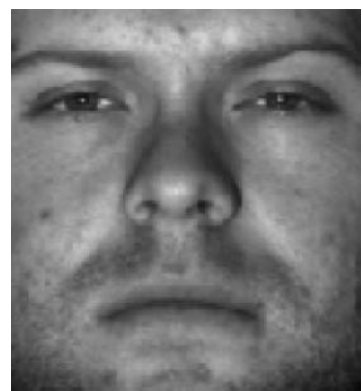

(a)

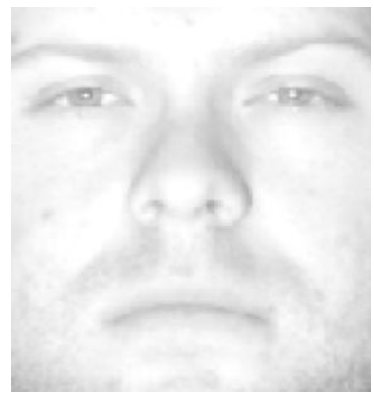

(b)
Fig.2(a) Original image(b) Preprocessed logarithmic image

\subsection{NSCT-based denoising model for illumination invariant extraction}

The stages to generate the NSCT are 1) 2-directional wavelet transform to generate $L L, H h$ and $H v$. 2) Finer directional decomposition of $H h$ and $H v$. 3) Iterate the one-level NSCT on $L L$. . All the NSCT sub bands are given as the following:

$\left\{\mathrm{Ci}_{0}, \mathrm{C}_{\mathrm{ij}}\right\}, \mathrm{i}, \mathrm{i}_{0}=1,2, \ldots \ldots, \mathrm{n} ; \mathrm{i} \leq \mathrm{i}_{0}$;

$\mathrm{j}=2,4,8 \ldots \ldots, \mathrm{m} ; \mathrm{n} \in \mathrm{N}, \mathrm{m} \in 2^{\mathrm{N}}$, where $\mathrm{i}$ is the scale of decomposition, $\mathrm{j}$ is the direction of decomposition, $\mathrm{C}_{\mathrm{i} 0}$ is the low frequency coefficient, $\mathrm{C}_{\mathrm{i}, \mathrm{j}}$ is the high frequency coefficient at the $\mathrm{j}$-th directional subband of the $\mathrm{i}$-th scale. In this model, $\mathrm{i}_{0}=3$ and $\mathrm{j}=\{2,4,8\}$.

The next step is to select a threshold for image denoising.

$t_{i j}=\frac{\beta \sigma_{i j}^{2}}{\sigma_{i j}^{x}}$

$\beta=\sqrt{\log \left(\frac{L_{i j}}{S}\right)}$

$\sigma_{i j}=\frac{\text { Median }\left|C_{i j}\right|}{\lambda}$

$\sigma_{i j}^{x}=\frac{1}{m \cdot n} \sum_{k=1}^{m} \sum_{l=1}^{n}\left(C_{i j}(k, l)-\overline{C_{l j}}\right)^{2}$

Where $\lambda$ is a parameter, whose general value is $0.6745, \mathrm{~m}$ and $\mathrm{n}$ are the height and width of an image, $\mathrm{L}_{\mathrm{ij}}$ is the length of the $\mathrm{j}$-th directional subband of the $\mathrm{i}$-th scale, $\mathrm{S}$ is the maximal scale of NSCT, and $C_{i j}$ is the average of coefficients at the $j$ th directional subband of the $\mathrm{i}$-th scale.

$c_{i j}^{t h r}=\left\{\begin{array}{cc}0 & \left|c_{i j}\right|<t_{i j} \\ c_{i j}-w t_{i j} & c_{i j} \geq t_{i j} \\ c_{i j}+w t_{i j} & c_{i j} \leq t_{i j} \\ t_{i j}\end{array}\right.$
$w=\frac{\left|c_{i j}(k, l)\right| \exp \left(\left|c_{i j}(k, l)\right|-t_{i j}\right)}{}$

The algorithm for extracting the illuminant variant features is given below.

1. Take the logarithm operation for the original face image $F$, $\mathrm{F}^{\prime} \approx=\log (\mathrm{F})$.

2. Perform multiscale decomposition of the logarithm facial image $\mathrm{F}^{\prime}$ by using NSCT.

3. Compute threshold $t_{\mathrm{ij}}$ at each directional subband of each scale by using Eqs.(4)-(7).

4. Apply soft thresholding to each directional subband of each scale by using Eqs.(8),(9).

5. Reconstruct illumination $\mathrm{I}^{\prime}$ from the modified NSCT coefficients by inverse NSCT.

The sample of illuminant invariant obtained through NSCT is shown in fig 3 .

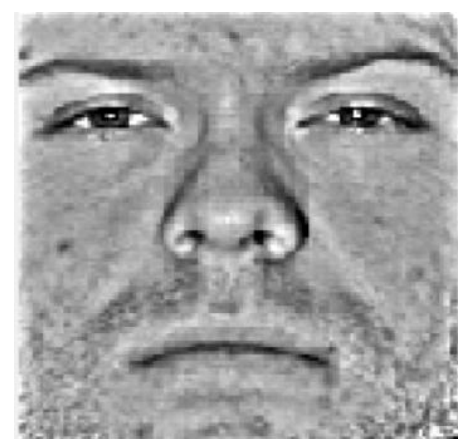

Fig.3 illuminant invariant

\subsection{ACO feature selection}

In an Artificial Ant Colony System developed by imitating the real ant's behavior, ant chooses its next step depending upon the transition probability matrix which is a function of the amount of pheromone on the path and the heuristic factor, e.g. the distance between the points. We now assume that there are $\mathrm{M}$ ants constituting an ant colony. Initially at $\mathrm{t}=0$, let the value of the pheromone present on each edge be $\tau_{i j}(0)$. In $\mathrm{K}$ iterations, each ant moves through a distance making a route. The pheromone deposited on every route is computed from:

$\tau_{\mathrm{ij}}(\mathrm{k}+1)=\rho \tau_{\mathrm{ij}}(\mathrm{k})+\Delta \tau_{\mathrm{ij}}(\mathrm{k}+1)$ 
$\tau_{\mathrm{ij}}(\mathrm{k}+1)=\left\{\begin{array}{c}\frac{\mathrm{Q}}{\mathrm{Lm}} \text { if ant uses edges } \mathrm{ij} \text { in its route } \\ 0\end{array}\right.$

where $0<\rho<1$ is a coefficient that represent the attenuation of trail. Q is a positive constant. Lm is the length of the route which an ant has traversed in its tour. The transition probability matrix, $\mathrm{P}_{\mathrm{ij}}(\mathrm{t})$ is given by:

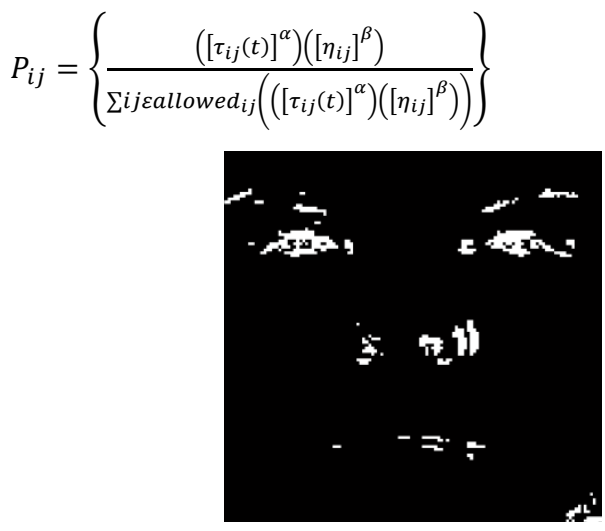

Fig 4 optimal feature extracted image

When $\eta_{i j}$ is the heuristic factor, allowed ${ }_{i j}$ is the set of points available, $\alpha$ and $\beta$ are two application dependent parameters that control the relative importance of the two main factors. $\tau_{i j}(\mathrm{t}) \mathrm{j}$ and $\eta_{\mathrm{i}}$. Transition probability matrix is obtained for every ant and those ants whose matrix remains null are not moved. When all ants have stopped moving it means that we have reached the minima. The corresponding output is shown in figure4.

\subsection{Graph Transformation Matching}

In this algorithm the relationship between adjacent features relies on the angular distances between neighboring features. Also, the matching between corresponding features in two images will proceed only if the angles between each feature and its neighboring features provide sufficient support to that matching. The angular distance is also less sensitive to the noise originating from lens distortion. Following steps describe the proposed algorithm.

1) Assume there are two sets of $N$-feature points with an initial one-to-one relationship $\left(P=\{p i\}\right.$ and $P^{\prime}=\left\{p i^{\prime}\right.$ \}). Two median $K-N N$ graphs $G_{P}=\left(V_{P}, E_{P}\right)$ (with adjacency matrix $A_{P}$ ) and $G_{P^{\prime}}=\left(V_{P^{\prime}}, E_{P^{\prime}}\right)$ (with adjacency matrix $A_{P^{\prime}}$ ) are generated for the sets $P$ and $P^{\prime}$ respectively. A vertex $v_{i}$ for each match correspondence $p i$, such that $V P=\{v 1, \ldots, v N\}$. A directed edge $(i, j)$ exists when $p j$ is one of the closest neighbors of $p i$ and also $\|p i-p j\| \leq \eta$. Here $\eta$ is defined by:

$\eta=\underset{(l, m \epsilon) V_{p} \times V_{p}}{\operatorname{median}}\left\|p_{l}-p_{m}\right\|$

2) Find all vertices of $G P$ with at most one edge (connection) with other vertices $\left.\left(\left\{v_{i} \mid \sum_{\forall_{j,(i, j)} \in \in_{E P}} A_{P(i, j}\right) \leq 1\right\}\right)$ and remove them and their correspondences from $G_{P}$ and $G_{P^{\prime}}$. Recompute both $G_{P}$ and $G_{P^{\prime}}$. Repeat this step until all vertices of $G P$ have minimum of two edges.

3) A weight matrix $W$ is generated for each point $p_{i}$ using graph $G_{P}$. For the edge that connects vertex $v_{i}$ to $v_{m}$ compute a weight value using the following equation:

$w_{(i . m)}=\left|\operatorname{Acos}\left(\frac{\left(p_{m}-p_{i}\right) \cdot\left(\left(p_{m-}^{\prime} p_{i}^{\prime}\right) \operatorname{Rot}\left(\theta\left(k_{m i n}, i\right)\right)\right)}{\left\|p_{m}-p_{i}\right\|\left\|p_{m-}^{\prime} p_{i}^{\prime}\right\|}\right)\right|$

where

$$
\operatorname{Rot}\left(\theta\left(k_{\text {min }}, i\right)\right)\left[\begin{array}{cc}
\cos \left(\theta\left(k_{\text {min }}, i\right)\right) & \sin \left(\dot{\theta}\left(k_{\text {min }}, i\right)\right) \\
-\sin \left(\theta\left(k_{\text {min }}, i\right)\right) & \cos \left(\theta\left(k_{\text {min }}, i\right)\right)
\end{array}\right]
$$

Here $k_{\min }$ represents the optimal rotation angle between each pair of matches. $p_{i}$ and $p_{m}$ are the $2 \mathrm{D}$ vectors of image coordinates for vertices $v i$ and $v_{m}$ and $A_{\text {cos }}$ is the inverse cosine function. The optimal rotation angle is defined as the angle that minimizes the sum of angular distances between vectors $v_{i}$ and $v_{m}$

$k_{\min }=$

$\underset{\forall k,(i, k) \epsilon E_{p}}{\operatorname{argmin}} \sum_{\forall j,(i . j) \epsilon E_{p}}\left|A \cos \left(\frac{\left(p_{j}-p_{i}\right) \cdot\left(\left(p^{\prime} j-p_{i}\right) \operatorname{Rot}(\theta(k, i))\right)}{\left\|p_{j}-p_{i}\right\|\left\|p^{\prime} j m-p \prime^{\prime}\right\|}\right)\right|$ And

$\theta(k, i)=\tan _{-\pi, \pi}\left(p_{k}-p_{i}\right)-\tan _{-\pi, \pi}\left(p_{k}^{\prime}-p_{i}^{\prime}\right)$

In the above equation $v$ is a 2D image vector with the $x$ and $y$ coordinates of $v_{x}$ and $v_{y}$.At this point, the weight matrix $W$ is filled by the angular distances between vectors of corresponding matched vertices and all of their connected vertices. All elements of $W$ that have not been set to any value are set to null.

4) For each vertex $v_{i}$, find the percentage of edges connected to $v_{i}$ with their correspondences connected to $v_{i}^{\prime}$.If the percentage is smaller than $50 \%$, the weight value of all different edges in the weighted matrix should be replaced by $\pi$.

$\forall i, j: W(i, j)=\pi$, if $\frac{\sum_{\forall k,(i, k) \in E_{p}} A_{p^{\prime}(i, k)}}{\sum_{\forall k,(i, k) \in E_{p}} A_{p(i, k)}}<0.5$

5) For each vertex $v_{i}$ of $G_{P}$, compute the mean of all weights related to all edges connected to it:

$w(i)=\underset{\forall j(i, j) \in E_{p}}{\operatorname{mean}}(W(i, j))$

Remove vertex corresponding to the maximum value of $w$ and all of its corresponding vertices from $P$ and $P^{\prime}$.

6) Find the maximum element of $W$ (wmax).

$w_{\max }=\max _{\forall i, j}(W(i, j))$

Set

$\mu_{\text {new }}=\underset{\forall i}{\operatorname{mean}(w(i))}$

If $w_{\text {max }}<\pi$ and $\left|\mu_{\text {new }}-\mu_{\text {old }}\right|<\epsilon$ stop; otherwise, set $\mu_{\text {old }}=\mu_{\text {new }}$ and proceed to the next iteration. The value of $\in$ is set to 0.001 (found empirically) for all the results presented in this work. The value of $\mu_{\text {old }}$ was set to $2 \pi$ initially. The graph obtained for test image and training image using GTM is shown in fig 5 .

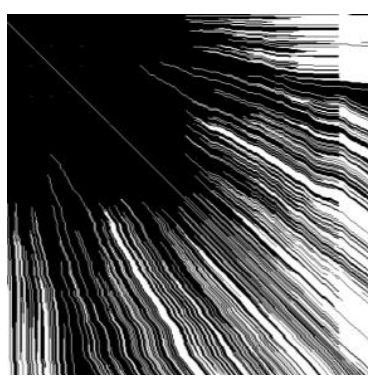

(a)

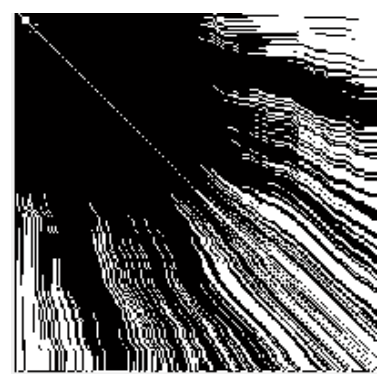

(b)
Fig 5 (a) Graph of the test image (b) Graph of the training image of database 2

\section{RESULTS AND DISCUSION}

For Experimental analysis, the frontal face image of 4 individuals of 50 different illuminations and various poses were taken from the well known yaleB database. In addition, the cropped images fall in to four subsets. One image per set is chosen as a training set and the rest as a testing set. The algorithm is implemented and tested for all the images. This ACO integrated illuminant invariant system detects the edges 
optimally in all the cases and it is found to be efficient. From the ACO output, it is obvious that the fine edges are detected in a better way and the feature subset get reduced. This optimal feature extracted image is then given to the graph matching algorithm which compares the graph pattern of the test image with the training image chosen randomly from each set. Finally the system retrieves a set of images which closely resemble the original image. To compare the performance, the results yielded by our method were compared with those obtained by sobel and Adaboost in terms of precision rate, recall rate, error rate and are given in table1. Also, the quality of results is quantitatively evaluated by recognition rates. As can be seen, the proposed method produces better natural images than sobel and adaboost methods. The results in fig 6 show that the proposed method is more robust to different illumination conditions than other conventional methods.

Table 1. Comparison of Various face recognition methods for low illuminant variant image in yale dataset

\begin{tabular}{|c|c|c|c|c|}
\hline Techniques & $\begin{array}{c}\text { Precision } \\
\text { rate }\end{array}$ & $\begin{array}{c}\text { Recall } \\
\text { rate }\end{array}$ & $\begin{array}{c}\text { Error } \\
\text { Rate }\end{array}$ & $\begin{array}{c}\text { Recog. } \\
\text { Efficiency }\end{array}$ \\
\hline Ada Boost & 0.75 & 0.85 & 0.250 & 0.85 \\
\hline Sobel & 0.8750 & 1 & 0.125 & 1 \\
\hline Proposed & 1 & 1 & 0 & 1 \\
\hline
\end{tabular}

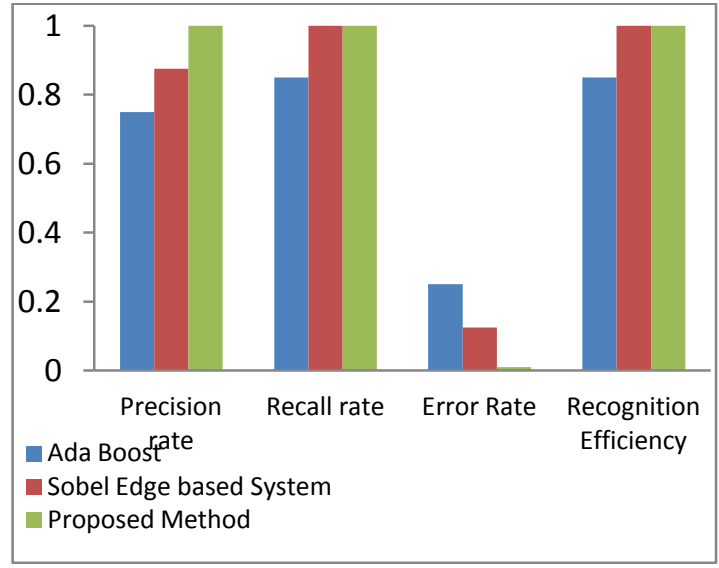

Fig. 6 Comparison of Various face recognition methods for low illuminant variant mage in yale dataset

\section{CONCLUSION}

In this paper, a simple and fast face detection system is proposed. The proposed method can extract illumination invariant features from the multiscale space optimally. For the images of low illumination and high illumination, this combined approach can recover the lost details with the enhancement of saturation only to some extent. Also the extracted geometrical structure is given to an adapted graph matching algorithm which retrieves the correct matches effectively. Experimental results on the well-known face database and also the comparative analysis shows that the proposed method is efficient and has better recognition efficiency and recall rate. Future work in this research work can be extended for rotation invariant, occluded images and more head poses.

\section{REFERENCES}

[1] Yong Cheng, Yingkun Hou, Chunxia Zhao, Zuoyong Li, Yong Hu, Cailing Wang 2010, "Robust face recognition based on illumination invariant in nonsubampled contourlet transform domain", Neurocomputing, pp. 2217-2224

[2] A. Amali Asha,S.P. Victor ,A. Lourdusamy 2011, "Feature Extraction in Medical Image using Ant Colony Optimization : A Study" , International Journal on Computer Science and Engineering Vol. 3 No. 2 ,pp.714-721

[3] Om Prakash Verma, Puneet Kumar, Madasu Hanmandlu, Sidharth Chhabra 2012, "High dynamic range optimal fuzzy color image enhancement using Artificial Ant Colony System", Applied Soft Computing,pp. 394-404.

[4] Graham Finlayson, Steven Hordley, Gerald Schaefer, GuiYun Tian 2005, "Illuminant and device invariant color using histogram equalization" Pattern Recognition, pp. $179-190$.

[5] Wen-chung Kao, Ming-chai Hsu, Yueh-Yiing Yang 2010, "Local contrast enhancement and adaptive feature extraction or illumination-invariant face recognition", Pattern Recognition,pp.1736-1747.

[6] W.shen, L.G.Yu, Y.L.Wang, J.Y.Yang,Z.W. Zhang 2012, "illumination invariant extraction for face recognition using neighboring wavelet coefficients" Pattern Recognition45,pp.1299-1305

[7] Dang-Hui Liu, Kin-Man Lam, Lan-Sun Shen 2005, “ Illumination invariant face recognition", Pattern Recognition ,pp.1705 - 1716.

[8] Young Kyung Park, Seok Lai Park, Joong Kyu Kim 2008, "Retinex method based on adaptive smoothing for illumination invariant face recognition" Signal Processing ,pp.1929-1945.

[9] Bolun Chen , Ling Chen , Yixin Chen 2012 , "Efficient ant colony optimization for image feature selection", SignalProcessing,pp.1-11.

[10] De-Sian Lu, Chien-Chang Chen 2007, "Edge detection improvement by ant colony optimization" Pattern Recognition ,pp.416-425.

[11] Hu Han , ShiguangShan , XilinChen, WenGaoc 2012, “A comparative study on illumination preprocessing in face recognition" Pattern Recognition,pp.1691-1699

[12] Mohammad Izadi and Parvaneh Saeedi 2012," "Robust Weighted Graph Transformation Matching for Rigid and Nonrigid Image Registration",IEEE Transactions on image processing, vol. 21, no. 10, pp.4369-4382.

[13] Hochul Shin , Seong-Dae Kim , Hae-Chul Choi 2007, “ Generalized elastic graph matching for face recognition" Pattern Recognition ,pp.1077-1082. 